\title{
High prevalence of $d h f r$ and dhps molecular markers in Plasmodium falciparum in pregnant women of Nchelenge district, Northern Zambia
}

\author{
Mwiche NP Siame ${ }^{1,3^{*}}$, Sungano Mharakurwa ${ }^{2}$, James Chipeta', Philip Thuma ${ }^{3}$ and Charles Michelo ${ }^{1}$
}

\begin{abstract}
Background: Sulphadoxine-pyrimethamine (SP) is the recommended drug for intermittent preventive treatment in pregnancy (IPTp) in most African countries, including Zambia. However, malaria is still one of the leading causes of morbidity and mortality in pregnant women despite reports of greater than 50\% of women taking at least two doses of SP in IPTp. Studies have shown that resistance to SP is associated with mutations in the dhfr and dhps gene of Plasmodium falciparum. This study examined the prevalence of $d h f r$ and dhps polymorphisms in P. falciparum found in pregnant women of Nchelenge district.

Method: This cross-sectional study was conducted in 2013 in Nchelenge, a holoendemic area with malaria prevalence estimated at 50\% throughout the year. Three rural health centres were randomly selected and a census survey carried out at each health centre. A questionnaire was administered and malaria testing done using RDT and microscopy, with collection of a dried blood spot. A chelex extraction was done to extract parasite DNA from dried blood spots followed by nested PCR and enzyme restriction digestion.
\end{abstract}

Results: Of the enrolled participants $(n=375)$, the median age of the women was 23 . The prevalence of malaria by PCR was $22 \%$. The PCR positive samples examined $(n=72)$ showed a high prevalence of $d h f r$ triple (Asn-108 + Arg-59 + lle-59) mutant (68\%) and dhps double (Gly -437 + Glu-540) mutant (21\%). The quintuple haplotype was found in 17\% with 2 samples with an additional Gly-581 mutation. In addition 6\% mutations at Val- 16 were found and none found at Thr-108 respectively, these both confer resistance to cycloguanil. Multivariate analysis showed that there was an association between malaria and women aged 30-34 years old $p<0.05$ (AOR: 0.36 ) at 95\% Cl.

Conclusion: This study showed a high number of mutations in the dhfr and dhps genes. The high malaria endemicity in the general population of this area may have contributed to the high prevalence of resistant parasites in pregnant women, suggesting a need to examine the efficacy of SP given that it is the only approved drug for IPTp in Zambia.

Keywords: Zambia, dhfr, dhps, Malaria in pregnancy, SP

\section{Background}

Malaria remains a major cause of death predominantly in sub-Saharan Africa. Statistics by the World Health Organization attribute an estimated 627,000 deaths to this disease in 2012 alone [1]. Children under five and pregnant women in high transmission and impoverished areas are at most risk of the disease. Over 30 million pregnant women are estimated to be at risk of Plasmodium

\footnotetext{
* Correspondence: siame_mwiche@yahoo.com

'Department of Public health, University of Zambia, School of Medicine, Lusaka, Zambia

${ }^{3}$ Macha Research Trust, Choma, Zambia

Full list of author information is available at the end of the article
}

falciparum malaria in sub-Saharan Africa annually [2]. The pathological effects of malaria adversely affect pregnancy outcome. The unborn child is at increased risk of premature and still-birth, low birth weight, congenital malaria, anaemia and impaired foetal growth [3-5]. Epidemiological settings and host immunity greatly influence the clinical presentation of pregnancy-associated malaria [6-9].

Zambia has achieved over 50\% reduction in malaria prevalence in the past decade [10]. However, the challenge remains, as malaria is still one of the leading causes of morbidity and mortality responsible for at least 36\% of hospital admissions and $20 \%$ of maternal mortality [11]. 
Sulphadoxine-pyrimethamine is the recommended drug by the World Health Organization for intermittent preventive treatment in pregnancy (IPTp) [12]. IPTp is made available through antenatal care (ANC) at government health facilities and an overall increase in access to ITNs, IRS and IPTp has been reported nationwide [13-15].

In Zambia, IPTp was adopted in 2003. It is administered presumptively three months after quickening (16-26 weeks) [16]. The pregnant woman is given one dose (each containing $500 \mathrm{mg}$ of sulphadoxine and $25 \mathrm{mg}$ pyrimethamine) for every ante natal care visit one month apart, under direct observation of the health worker [17]. Prior to the universal adoption of SP in IPTp randomized controlled trials have showed that SP supplemented with iron decreases the risk of placental parasitaemia, maternal anaemia and low birth weight [18-20].

Drug resistance is one of the greatest challenges of malaria control programmes. Hence, while SP remains ideal for IPTp, in stable transmission areas, increasing resistance has been reported [21,22]. SP resistance is linked to point mutations in the parasite genome specifically the dihydrofolate reductase (dhfr) and dihydpteroate synthetase (dhps) genes. Mutations in $d h f r$ confer resistance to pyrimethamine while mutations in dhps confer resistance to sulphadoxine and other sulpha drugs. There are variations in SP mutations, single, double or triple - the more mutations, the stronger the resistance. The dhfr triple mutant (Asn-108 + Ile-51 + Arg-59) and dhps double mutant (Gly-437 + Glu-540) have been strongly associated with potential resistance in subSaharan Africa [23,24].

A study done in 2006 in six districts in the general population of Zambia showed variation of rates of resistance to SP with mutated $d h f r$ frequency ranging from $71-92 \%$ and $39-71 \%$ frequency for the double mutant dhps, respectively [25]. Therefore, there is a need for close monitoring and surveillance of the efficacy of SP in pregnant women. This study aimed to investigate the presence of malaria in pregnancy, associated risk factors and determine the prevalence of molecular markers that confer resistance to SP in a highly endemic area.

\section{Methods}

\section{Design and sampling procedures}

This cross-sectional study was conducted in Nchelenge district, Luapula province, Zambia in February to April 2013. The population of Nchelenge is estimated at 180,000 and the inhabitants are mostly fishermen and peasant farmers. Nchelenge has one first level referral hospital St Paul's Mission hospital, ten rural health centres and three health posts. This is a holoendemic area of malaria transmission with an estimated 50\% confirmed cases throughout the year, hence transmission is throughout the year. An estimated $80 \%$ of recorded deaths are attributed to malaria with the most affected being children under five years of age and pregnant women. The target population of this study was pregnant women who were residents of Nchelenge and attended antenatal care at any of the three health facilities selected for the study (Table 1). Parental consent and child assent was obtained for those below 18 years old. Non-pregnant women, pregnant women not resident in the study sites or those within the catchment area but severely ill, were not eligible to be included in the study.

Multi-stage sampling was employed. The district was conveniently selected while simple random sampling using Microsoft Excel was used to select the three rural health centres (RHCs) out of 14 in the district and these three were the sites chosen for the study. The rural health centres selected were Kabuta, Nchelenge and Kashikishi RHC. Consenting individuals were included in a census survey from the rural health centres. Hence, all pregnant women who came to the centre for antenatal care, regardless of whether for re-visits or first attendance, were eligible to be included in the study.

The prevalence of malaria in pregnant women was assumed to be $50 \%$, which is the prevalence for the general population in Nchelenge. The calculated total sample size was 384 with a power of 80 at $95 \%$ CI.

\section{Data collection}

The data collection tool used in this study was a structured questionnaire to document demographic data and risk factors for the presence of malaria. To validate the data collection tool pre-testing of the questionnaire was done in two ways: the tool was pre-tested to ensure clarity of questions in English and then for consistency, precision, clarity of questions and correct translations in Bemba. Necessary adjustments and revisions were made based on the pre-test results.

\section{Laboratory methods}

The eligible pregnant women were screened for malaria and anaemia on the scheduled ante-natal care day. Malaria was defined as a positive laboratory test for $P$. falciparum

Table 1 General characteristics of pregnant women of Nchelenge district $(n=375), 2013$

\begin{tabular}{ll}
\hline Characteristic & $\mathbf{n}$ or $\%$ \\
\hline Median age & 23 years \\
Median gestational stage & $6-9$ months \\
Primigravidae & $24 \%$ \\
Multigravidae & $54 \%$ \\
Married & $86 \%$ \\
No SP intake & $15 \%$ \\
$>1$ dose of SP & $64 \%$ \\
\hline
\end{tabular}


(RDT and/or smear and/or PCR). Approximately 100 microlitres of venous blood was collected from each participant by a single finger prick. A rapid diagnostic test (RDT) and microscopy was used to test for malaria. The RDT used detects the antigen histidine rich protein II (HRP II) found on the parasite surface, the test kits brand was the Standard Diagnostics Bioline Malaria antigen Pf test (SD-p. $\mathrm{f}^{\mathrm{TM}}$ ) manufactured by Standard Diagnostics Inc., South Korea. The test results were ready within 15 minutes. Thick blood smears were collected on microscope slides for later reading by a microscopist.

Dried blood spots (DBS) were also collected on Whatman $3 \mathrm{MM}$ filter paper at the same time as the RDT and thick smear. Plasmodium falciparum DNA was extracted from the DBS by a chelex protocol and amplified by polymerase chain reaction (PCR) followed by restriction enzyme digestion. Mutations were defined as the detection of polymorphisms in the dhfr gene specifically at Asn-108, Arg-59, Ile-59 and dhps Gly-437, Glu-540 and Gly-581 mutation. Mixed infections were those that showed both sensitive and resistant parasite strains while sensitive strains were parasites that showed no polymorphisms in the region of interest after restriction digestion. Quality assurance was done at Tulane University, School of Public Health and Tropical Medicine in the Kumar Laboratory. Positive samples $(n=10)$ were randomly selected and PCR was done followed by restriction digestion with enzymes BStNI, AluI and BSrI. The results were similar to those initially obtained hence results were reproducible and reliable.

\section{Data analysis}

Analysis was done using Stata version 12.2. Variables were defined, descriptive statistics; means, medians, standard deviations and odds ratios were used to assess the data. The factors examined were age, marital status, gravidity, education level, ITN ownership and usage, malaria knowledge, medical history and SP intake. These were self reported and SP intake was confirmed by medical records. Chi square was used to determine the association between malaria and each of the associated factors. Significance (p-value) was set at $5 \%$ with a confidence interval at 95\%. Multivariate analysis using logistic regression was done for adjustments, to control for confounding and to examine the odds ratios for malaria prevalence in relation to each of the risk factors.

\section{Ethics}

Ethical approval was sought from the University of Zambia Biomedical Research Ethics Committee (UNZABREC) and granted in February, 2013 (IRB004-11-12).

\section{Results}

\section{Population characteristics}

A total number of 400 participants were enrolled in this study, of these 375 had complete data. The median age of the participants was 23 years old (IQR 20-24). The median gestational stage was 24-36 weeks. Of the women enrolled $57 \%$ reported to have had a primary education while only $3.2 \%$ reported a college/university attainment. Of these women $15 \%$ had not received any SP at the time of enrollment. The major source of nonparticipation was refusal $(<5 \%)$ and incomplete data.

\section{Malaria infection risk factors}

The prevalence of malaria by rapid diagnostic test was $30 \%$, by microscopy prevalence was $15 \%$ and $22 \%$ by PCR. The rapid diagnostic test is an antigen-based test and may remain positive two weeks after treatment, hence may result in false positives. Using Chi square analysis, significant results were obtained for malaria symptom knowledge and age. Over $75 \%$ of women with symptom knowledge of malaria had taken medication in the last malaria infection, with the greatest proportion $(50 \%)$ having taken artemether-lumefantrine $\left(\right.$ Coartem $\left.^{\odot}\right)$. In terms of insecticide-treated net distribution, $74 \%$ of the women reported to own at least one net in their household although the results suggest that there was no association with malaria. Using multivariate analysis none of the factors were significantly associated with malaria except age. Women aged 30-34 were 64\% less likely to have malaria compared to those in the reference group 14-18 years old (AOR: 0.36 95\% CI).

\section{Genotyping}

Of the PCR positives, 72 were genotyped using restriction fragment length polymorphism (RFLP) for the presence of mutations at the different loci. The prevalence of $d h f r$ and dhps mutations are shown in Figures 1 and 2. The prevalence of the dhfr triple mutant (Asn-108 + Ile-51 + Arg-59) was $68 \%$ and dhps double mutant (Gly-437 + Glu-540) was $21 \%$. The quintuple mutant was present in $17 \%(n=12)$ of the samples and the sextuple mutant was present in $3 \%$ $(\mathrm{n}=2)$ of the samples. The figures show additional codons at Val-16 and dhps mutation at codon 436. There was no association between SP intake and genotypes present.

\section{Discussion}

This study documents high malaria prevalence and antifolate resistance mutations in pregnant women in a holo endemic area. It is important to note that $>60 \%$ of women who were found with malaria had taken at least one dose of SP. However, with multivariate analysis there was no association between presence of malaria and any of the associated risk factors except age, hence the association found with 


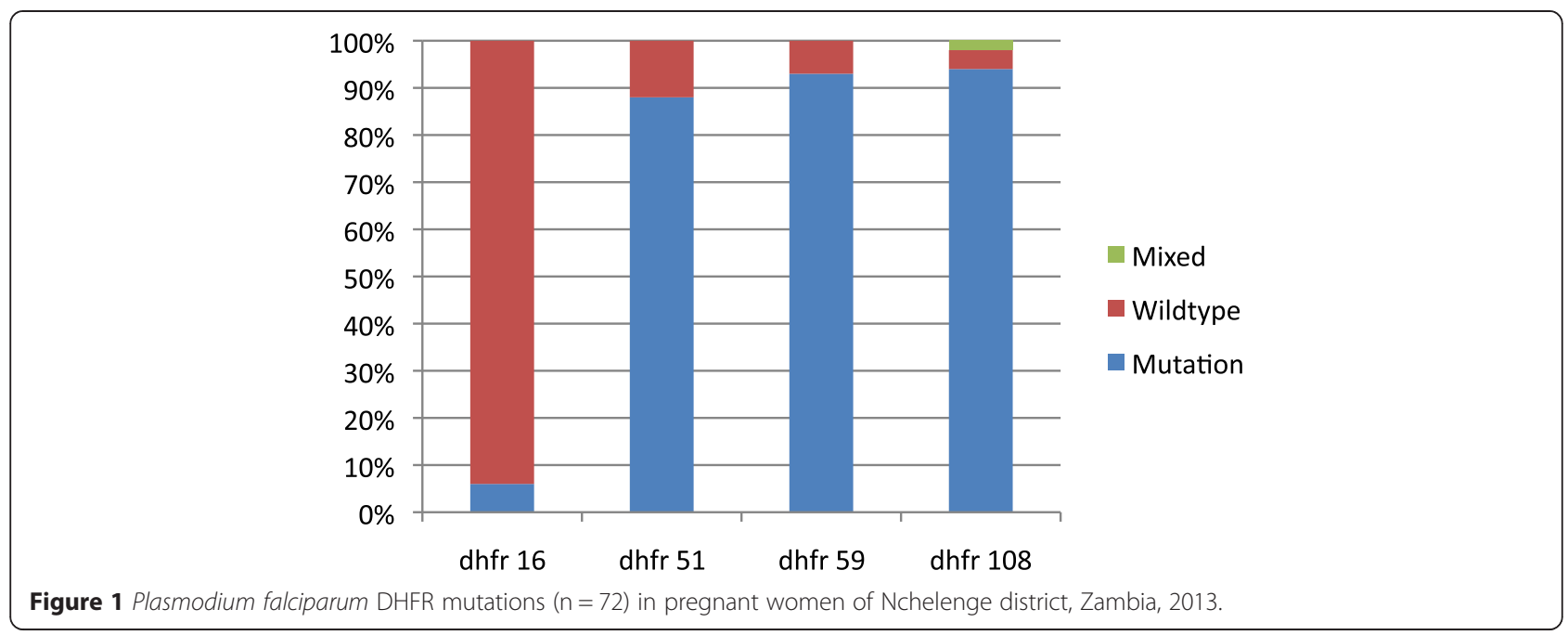

chi square may be due to confounding. The multivariate analysis on predictors of malaria prevalence suggests an association between malaria prevalence and women aged 30-34 years compared to those women aged 14-18 years old. This may be due to a protective effect reported in other studies that older women who are likely to have had more than one previous pregnancy (multigravidae) are less likely to have malaria compared to those who have not had children previously (primigravidae) [25].

Possible bias due to non-participation may have occurred as this study was based exclusively at the health centres thereby excluding those pregnant women who did not attend antenatal clinic and who may have a different profile from those that do. Another study limitation was that clinical efficacy of SP was not determined in order to assess treatment failure, and the data collected including when the last malaria medication was taken may be subject to recall bias.
The results obtained show high levels of dhfr mutations indicating mostly pyrimethamine resistance but also a higher emergence of mutations Val-16 which is an indication of resistance to cycloguanil and a higher emergence of mutations at dhps 581 than previously reported. Another study conducted in Mansa, Zambia in pregnant women showed $63 \%$ had quintuple mutants while only two had an additional mutation at the Gly581 loci. The treatment failure recorded in the study was $22 \%$. This shows that the dhps mutations are becoming more common and there has been an escalation in the mutations in this population. This is consistent with other studies that have shown that in hyperendemic areas with high rate of transmission as is the case for Nchelenge, resistant parasites have a tendency to increase and contribute to treatment failure [26]. SP has been in use for over two decades in Zambia and due to drug pressure parasite resistance is expected to be high

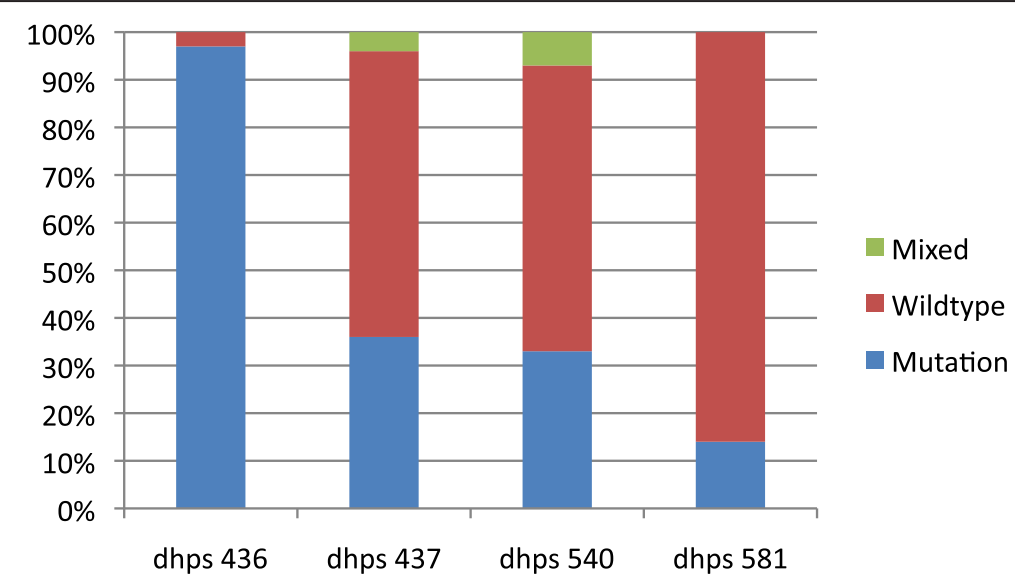

Figure 2 Plasmodium falciparum dhps mutations $(n=72)$ in Plasmodium falciparum parasites in pregnant women of Nchelenge district Zambia, 2013. 
as is evident from trends analysis in populations were SP has been used for even shorter periods of time. Data from southern Zambia showed that by 2006, the prevalence of $d h f r$ and dhps mutants had escalated rapidly since 1988, and that the quintuple (dhfr triple $+d h p s$ double) mutant associated with highest levels of SP clinical failure was starting to be seen (6.5\%) [27].

Studies done in Tanzania have shown an emergence of the presence of the dhps triple mutant and suggested that IPTp with SP may not improve overall pregnancy outcome [28]. Furthermore conflicting results have been found with some studies showing increased prevalence of $d h f r$ and dhps mutations while still others have found that SP IPTp is effective $[29,30]$.

High prevalence of multiple mutants raises concerns as these have been shown to correlate with therapeutic or clinical failures. Therefore there is need to explore other drugs as options for IPTp.

\section{Conclusion}

This study shows a high prevalence of malaria in pregnant women of Nchelenge district, despite reported use of SP-IPTp. This may be due to the high parasite resistance mutations to the drug as was evident in this study. The research implications are that there is need to conduct further high powered studies to determine efficacy and parasite clearance rates using SP-IPTp and also begin to explore alternative avenues for drugs to be used in IPTp if it is to be beneficial to this population.

\section{Competing interests}

The authors declare that they have no competing interests.

\section{Authors' contributions}

MS was responsible for the study design, carrying out the molecular work, field work and initiating and writing up the work. JC and PT contributed to the study design, the write up and data analysis and SM gave overall supervision of the lab work and analysis of molecular results. CM was the overall supervisor for this work and contributed to the protocol write-up, data analysis and manuscript write-up. All authors read and approved the final manuscript.

\section{Acknowledgements \\ Deepest gratitude to all the participants and all members of staff at Macha Research Trust, St Pauls Mission Hospital, Nchelenge Rural Health Centre, Kashikishi and Kabuta Rural Health Centres. Many thanks to Dr Matakala and Rogers Mwila for their valuable input into this work. This work was supported by Norad's Programme for Master studies (NOMA), The forgarty international institutes of health training grant, -the malaria training and capacity building in Southern Africa (MTCBSA: 2D43TW001587-06A2) and the Division of Microbiology and Infectious Diseases, National Institutes of Allergy and Infectious Diseases, National Institutes of Health as part of the International Centers of Excellence for Malaria Research (U19 Al089680).}

\section{Author details}

${ }^{1}$ Department of Public health, University of Zambia, School of Medicine, Lusaka, Zambia. ${ }^{2} J o h n s$ Hopkins Bloomberg School of Public Health, Baltimore, USA. ${ }^{3}$ Macha Research Trust, Choma, Zambia.

Received: 21 November 2014 Accepted: 4 April 2015

Published online: 06 May 2015

\section{References}

1. WHO. World malaria report 2013. Geneva: World Health Organization; 2013. http://www.who.int/malaria/publications/world_malaria_report_2013/ wmr2013_no_profiles.pdf?ua=1 last accessed 19th April, 2014.

2. World Health Organization/UNICEF. Africa malaria report 2003. WHO/CDS/ MAL/2003.1093. Geneva, Switzerland: World Health Organization.

3. Brabin BJ. The risks and severity of malaria in pregnant women, Applied field research in malaria reports no. 1. Geneva, Switzerland: World Health Organization; 1991

4. Verhoeff FH, Brabin BJ, van Buuren S, Chimsuku L, Kazembe P, Wit JM, et al. An analysis of intra-uterine growth retardation in rural Malawi. Eur J Clin Nutr. 2001;55:682-9.

5. Saba N, Sultana A, Mahsud I. Outcome and complications of malaria in pregnancy. Gomal J Med Sci. 2008;6:98-101.

6. Recker M, Bouma MJ, Bamford P, Gupta S, Dobson AP. Assessing the burden of pregnancy associated malaria under transmission settings. Malar J. 2009:8:245.

7. Steketee RW, Nahlen BL, Parise ME, Menendez C. The burden of malaria in pregnancy in malaria endemic areas. Am J Trop Med Hyg. 2001;64(1-2 Suppl):28-35.

8. Mount AM, Mwapasa V, Elliott SR, Beeson JG, Tadesse E, Lema VM, et al. HIV infection impairs humoral immunity to Plasmodium falciparum malaria in pregnancy. Lancet. 2004:363:1860-7.

9. Verhoeff FH, Brabin BJ, Hart CA, Chimsuku L, Kazembe P, Broadhead RL. Increased prevalence of malaria in HIV-infected pregnant women and its implications for malaria control. Trop Med Int Health. 1999;4:5-12.

10. WHO. Malaria deaths decline by $66 \%$ in Zambia. News release 2009. http:// www.who.int/mediacentre/news/releases/2009/malaria_deaths_zambia_ 20090423/en/ last accessed 24 April 2014.

11. National Malaria Control Center. Malaria control in Zambia. http:// www.nmcc.org.zm/malaria_control.html last accessed 24 April 2014

12. National Malaria Control Center. Prevention during pregnancy. http://www.nmcc.org.zm/prevention_during_pregnancy.htm last accessed 20th November 2014.

13. Updated WHO Policy recommendation on IPTp, Geneva October, 2012 http://www.who.int/malaria/iptp_sp_updated_policy_recommendation_ en_102012.pdf last accessed 20th November 2014.

14. Guidelines for the diagnosis and treatment of malaria in Zambia, Ministry of health, Government of the Republic of Zambia; 2010.

15. Ministry of Health. Malaria indicator survey report, Zambia 2010. http:// www.nmcc.org.zm/files/FullReportZambiaMIS2010_001.pdf last accessed 3rd May 2015.

16. Guidelines for the diagnosis and treatment of malaria in Zambia, fourth edition, Government of the Republic of Zambia, Ministry of Health guidelines 2014. http://www.nmcc.org.zm/files/GuidelinesonDiagnosisand TreatmentofMalariainZambia_4thEd_2-24-14.pdf last accessed on 3rd May 2015

17. McGready R, White NJ, Nosten F. Parasitological efficacy of antimalarials in the treatment and prevention of falciparum malaria in pregnancy 1998 to 2009: a systematic review. BJOG. 2011;118:123-35.

18. Kayentao KM, Newman RD, Maiga H, Doumtabe D, Ongoiba A, Coulibaly D, et al. Comparison of intermittent preventive treatment with chemoprophylaxis for the prevention of malaria during pregnancy in Mali. J Infect Dis. 2005;191:109-16.

19. Njagi JKMP, Estembale B, Ouma J, Mugo B. Prevention of anemia in pregnancy using insecticide-treated bednets and sulfadoxinepyrimethamine in a highly malarious area of Kenya: a randomized controlled trial. Trans R Soc Trop Med Hyg. 2003;97:277-82.

20. Schultz LJSR, Macheso A, Kazembe P, Chitsulo L, Wirima JJ. The efficacy of antimalarial regimens containing sulfadoxine-pyrimethamine and/or chloroquine in preventing peripheral and placental Plasmodium falciparum infection among pregnant women in Malawi. Am J Trop Med Hyg. 1994;51:515-22.

21. Nnaemeka C, Shah M, Gatei W, Van Eyk AM, Ayisi J, Kanuki S, et al. Temporal trends of sulphadoxine-pyrimethamine (SP) drug resistance molecular markers in Plasmodium falciparum parasites in Western Kenya. Malar J. 2012;11:34.

22. Mockehaupt FP, Bedu-Addo G, Eggeltte TA, Hommerich L, Holmberg V, Von Oertzen C, et al. Rapid increase in the prevalence of sulphadoxinepyrimethamine resistance among Plasmodium falciparum isolated from pregnant women in Ghana. J Infect Dis. 2008;198:1545-9. 
23. Duraisingh MJ, Curtis J, Warhurst DC. Plasmodium falciparum detection of polymorphisms in the dihydrofolate reductase and dihydropteroate synthetase genes by PCR and restriction digestion. Exp Parasitol. 1998;89:1-8.

24. Triglia T, Menting JG, Wilson C, Cowman F. Mutations in dihydropteroate synthase are responsible for sulfone and sulphanomide resistance in Plasmodium falciparum. Proc Natl Acad Sci U S A. 1997;94:13944-9.

25. Akanbi OM, Odaibo AB, Ademowo AG. The burden of malaria infection on pregnant women and birth weight of infants in south western Nigeria. East Afr J Public Health. 2009;6:63-8.

26. Tan KR, Katalenich BL, Mace KE, Nambozi M, Taylor SM, Wiegand RE, et al. Efficacy of Sulphadoxine-pyrimethamine for intermittent preventive treatment of malaria in pregnancy, Mansa, Zambia. Malar J. 2014;13:227.

27. Mkulama MA, Chishimba S, Sikalima J, Rouse P, Thuma P, Mharakurwa S. Escalating Plasmodium falciparum antifolate drug resistance mutations in Macha, rural Zambia. Malar J. 2008;7:87.

28. Gesase S, Gosling RD, Hashim R, Ord R, Naidoo I, Madebe R, et al. High resistance of Plasmodium falciparum to sulphadoxine/ pyrimethamine in northern Tanzania and the emergence of dhps resistance mutation at Codon 581. PLoS One. 2009;4, e4569.

29. Chanda PHM, Mharakurwa S, Shinondo C, Roper C, Pota H. Frequency of plasmodium falciparum dihydrofolate reductase and synthase resistance markers in six districts in Zambia. Med J Zambia. 2007;34:58-61.

30. ter Kuile FO, van Eijk AM, Filler SJ. Effect of sulfadoxine-pyrimethamine resistance on the efficacy of intermittent preventive therapy for malaria control during pregnancy: a systematic review. JAMA. 2007;297:2603-16.

\section{Submit your next manuscript to BioMed Central and take full advantage of:}

- Convenient online submission

- Thorough peer review

- No space constraints or color figure charges

- Immediate publication on acceptance

- Inclusion in PubMed, CAS, Scopus and Google Scholar

- Research which is freely available for redistribution 